\title{
PAX5-induced upregulation of IDH1-AS1 promotes tumor growth in prostate cancer by regulating ATG5-mediated autophagy
}

Nan Zhang ${ }^{1}$, Zhongyi Li', Fuding Bai ${ }^{1}$ and Shigeng Zhang ${ }^{1}$

\begin{abstract}
Prostate cancer ( $\mathrm{PCa}$ ) is one of the major malignancies affecting males' health around the world. Long noncoding RNAs (IncRNAs), a class of long transcripts, has been reported as essential regulators in tumorigenesis. IDH1 antisense RNA 1 (IDH1-AS1) is an IncRNA which can interact with genes to regulate the Warburg effect. However, function and mechanism of it in tumorigenesis of PCa remains unclear. Therefore, our current study focused on exploring the role of IDH1-AS1 in PCa tumor growth. At first, the expression of IDH1-AS1 was identified to be upregulated in PCa samples and cell lines. Mechanism associated with the upregulation of IDH1-AS1 was analyzed and demonstrated by mechanism experiments. The result suggested that PAX5 is the transcriptional activator of IDH1-AS1. Functionally, lossof function assays revealed that silencing of IDH1-AS1 inhibited cell proliferation and induced cell apoptosis both in vitro and in vivo. Through microarray analysis and Gene ontology (GO) analysis, we determined that IDH1-AS1 can affect PCa cell autophagy by upregulating ATG5 expression. Mechanism investigation further validated that IDH1-AS1 posttranscriptionally regulated ATG5 expression by enhancing the mRNA stability of ATG5 or upregulating ATG5 by sequestering miR-216b-5p. Consequently, rescue assays demonstrated that IDH1-AS1 promoted proliferation and apoptosis in PCa via ATG5-induced autophagy. Taken together, our study elucidated the function and regulatory mechanism of IDH1-AS1, thus providing a novel biomarker for PCa.
\end{abstract}

\section{Introduction}

Prostate cancer $(\mathrm{PCa})$, one of the most common diagnosed male malignancies ${ }^{1}$. Lacking of diagnostic biomarker in early stage is the main cause of low overall survival rate of patients with PCa. Despite the efforts in exploration of novel therapeutic strategies, patients' prognosis remains unfavorable. Thus, investigation of molecular mechanism involved in the tumorigenesis of $\mathrm{PCa}$ is essential to finding novel diagnostic biomarkers.

Long noncoding RNAs (lncRNAs), are a type of transcripts with length over $200 \mathrm{nt}$, which have become the research focus in cancer-associated transcriptome ${ }^{2}$. Functionally, lncRNAs can serve as biological participants

Correspondence: Shigeng Zhang (shigaishen2074@163.com)

'Department of Urology, The Second Affiliated Hospital Zhejiang University School of Medicine, No.88 Jiefang Road, 310009 Hangzhou, China

Edited by B. Zhivotovsky in diverse processes ${ }^{3,4}$. In addition, IncRNAs have been characterized as oncogenes or tumor suppressors in tumorigenesis ${ }^{5-7}$. Recently, PCa-specific IncRNAs were also defined ${ }^{8,9}$. In this study, we screened lncRNAs associated with PCa from online database. IDH1 antisense RNA 1 (IDH1-AS1) is a lncRNA that was differentially expressed in PCa samples obtained from database or 62 PCa patients. Xiang $\mathrm{S}$ et al. has reported the potential function of IDH1-AS1 in cancer growth ${ }^{10}$. Nevertheless, it is unclear whether IDH1-AS1 exerted function in PCa tumor growth via regulating molecular mechanism.

Transcription activation can induce upregulation of lncRNAs, thus promoting tumorigenesis ${ }^{11-13}$. Here, we also detected the transcription regulator of IDH1-AS1 through bioinformatics analysis and mechanism investigation. Moreover, loss-of function study was carried out in $\mathrm{PCa}$ cells to determine the role of IDH1-AS1 in 
regulating $\mathrm{PCa}$ cell growth both in vitro and in vivo. Downstream molecular mechanism of IDH1-AS1 was analyzed using microarray analysis and GO analysis. Then, we further detected whether IDH1-AS regulated PCa cell growth via ATG5-induced autophagy. The regulatory pattern of IDH1-AS1 in ATG5 was assessed by mechanism experiments.

LncRNAs are acknowledged to act as posttranscriptional regulators in tumorigenesis by serving as competing endogenous $\mathrm{RNAs}^{14-16}$ or interact with RNA-binding proteins to enhance mRNA stability ${ }^{17-20}$. In this regard, we investigated the posttranscriptionally regulatory effect of IDH1-AS1 on ATG5. Collectively, the focus of this study is on exploring the role of PAX5-activated IDH1AS1 in PCa tumor growth via ATG5-induced autophagy.

\section{Materials and methods}

\section{Bioinformatics analysis}

The expression pattern of IDH1-AS1 in human normal tissues and PCa tissues was downloaded from TCGA dataset (http://gepia.cancer-pku.cn/index.html). The DNA motif of PAX5 and its putative binding sites within IDH1-AS1 promoter were predicted via JASPAR tool (http://jaspar.genereg.net/).

\section{Patients and clinical specimens}

A total of 62 pairs of $\mathrm{PCa}$ and corresponding paracancerous tissues were randomly acquired from patients who underwent surgical resection at the Second Affiliated Hospital Zhejiang University School of Medicine from January 2012 to December 2018. All experimental subjects had not received any other treatment prior to operation. Written informed consents were signed by all subjects for study purpose. Our study was approved and supervised by The Ethics Committee of the Second Affiliated Hospital Zhejiang University School of Medicine.

\section{Cell lines and culture}

Human prostate epithelial cell line (RWPE-1) and four acknowledged human PCa cell lines (PC-3, DU145, VCaP, and $\mathrm{LNCaP}$ ) were all acquired commercially from the American Type Culture Collection (ATCC, Manassas, VA, USA). All cell lines were kept in RPMI 1640 medium (Gibco-BRL, Carlsbad, CA) with 10\% fetal bovine serum. Cell culture was performed in a humidified incubator with $5 \% \mathrm{CO}_{2}$ at $37^{\circ} \mathrm{C}$.

\section{RNA isolation and quantitative real-time PCR (qRT-PCR)}

At first, total RNAs were isolated from cells using Trizol reagent (Invitrogen, Carlsbad, CA, USA) following the user guide. Reverse transcription was conducted using the Applied Biosystems ${ }^{\text {Tw }}$ High-Capacity cDNA Reverse Transcription Kit. SYBR ${ }^{\bullet}$ Green Real-Time PCR Master
Mixes (Thermo Fisher Scientific, Waltham, MA, USA) was used to prepare PCR amplification reaction with GAPDH as endogenous control. For detecting miR-216b$5 \mathrm{p}$ level, miRNA was isolated using the mirVana miRNA Isolation Kit (Thermo Fisher Scientific), reverse transcription was conducted by the miScript II RT Kit (QIAGEN, Inc., Valencia, CA, USA), and PCR reaction was prepared by the mirVana qRT-PCR miRNA Detection Kit (Thermo Fisher Scientific) with U6 as endogenous control. All targets and references were amplified in triplicate. The relative expression levels of targets were calculated using $2^{-\triangle \triangle \mathrm{Ct}}$ method.

\section{Cell transfection and plasmids}

$\mathrm{VCaP}$ and LNCaP cell lines were cultivated all night to reach $70-80 \%$ confluence. All cell transfections were operated by use of lipofectamine2000 (Thermo Fisher Scientific). The pcDNA3.1 vector expressing PAX5, PTBP3, or ATG5 and empty vector (named as Ctrl) were designed and constructed by Sangon (Shanghai, China). To knockdown the expression of PAX5 or IDH1-AS1, the short hairpin RNAs (shRNAs) against PAX5 or IDH1-AS1 (named as shPAX5 or shIDH1-AS1\#1/2) and non-specific shRNA (named as shCtrl) were synthesized by GenePharma (Shanghai, China). MISSION ${ }^{\circledR}$ microRNA Mimics miR-216b-5p and scrambled negative control (named as miR-NC) were from Sigma-Aldrich (St. Louis, MO, USA). After $48 \mathrm{~h}$ of incubation, cells were reaped from three independent experiments and used for subsequent analysis.

\section{Luciferase reporter assay}

IDH1-AS1 promoter was amplified by PCR and subcloned into the pGL3-Basic vector (Promega, Madison, WI, USA), VCaP and LNCaP cell lines were seeded in 96-well culture plates (8000 cells/well) and co-transfected with reporter plasmids containing IDH1-AS1 promoter, pRL-TK-Renilla plasmid (Promega, USA) and pcDNAPAX5 or shPAX5, along with their respective control (Ctrl or shCtrl). After $48 \mathrm{~h}$, luciferase activity was measured using the Dual-Luciferase Reporter Assay System (Promega, USA). For assessing the interaction between miR216b-5p and IDH1-AS1 or ATG5, cells were cotransfected with miR-216b-5p mimics or miR-NC, together with pmirGLO-IDH1-AS1-WT/Mut vector (Sangon, China) or psiCheck2-ATG5-WT/Mut vector (Generay Biotech, Shanghai, China). Biological replications were conducted for three times.

\section{Chromatin immunoprecipitation (ChIP) assay}

ChIP assay was conducted by use of the EZ ChIP ${ }^{\mathrm{m}}$ Chromatin Immunoprecipitation Kit for cell line (Millipore, Bedford, MA, USA). $\mathrm{VCaP}$ and $\mathrm{LNCaP}$ cell lines were first fixed in $1 \%$ formaldehyde for 15 min crosslink. 
Thereafter, crosslinked chromatin was ultra-sonicated into 200- to 1000-bp fragments, and then immunopreciated with anti-PAX5 or control anti-IgG bound-protein $\mathrm{G}$ beads for $6 \mathrm{~h}$. qRT-PCR was used to evaluate the enrichments of DNA fragments immunoprecipitated by antibodies. ChIP assay was performed in triplicate.

\section{Cell proliferation assays}

$\mathrm{VCaP}$ and $\mathrm{LNCaP}$ cell lines were seeded into 96-well plates $\left(1.0 \times 10^{3}\right.$ cells/well $)$ in triplicate. Ten microliters of the Cell Counting Kit-8 (CCk-8, Dojindo, Kumamoto, Japan) was added into each well and incubated for $2 \mathrm{~h}$ in line with the user guide. Cell ability was examined by measuring the absorbance at $450 \mathrm{~nm}$ every $24 \mathrm{~h}$ for 4 days.

For colony formation assay, cells were seeded at a density of 500 cells per well into six-well plates, followed by incubation for two weeks. The colonies were dyed with $0.5 \%$ crystal violet solution for $15 \mathrm{~min}$ and finally counted.

For EdU incorporation assay, the Cell-light ${ }^{\mathrm{mm}}$ EdU ApolloR567 In Vitro Imaging Kit was purchased from Ribobio (Guangzhou, China). Cells were transfected and planted into 96-well culture plates (8000/well) all night. After 2 days, cells were cultivated with $25 \mu \mathrm{M}$ of Edu for $4 \mathrm{~h}$ and fixed in $4 \%$ paraformaldehyde for $30 \mathrm{~min}$, following permeabilizing with $0.5 \%$ TritonX-100 for $10 \mathrm{~min}$. DAPI was utilized to stain the total cells. All proliferative assays were repeated independently for at least three times.

\section{Cell apoptosis assays}

Cell apoptosis was measured by JC- 1 assay. $\mathrm{VCaP}$ and LNCaP cell lines were put into 96-well culture plates at the density of $1.0 \times 10^{4}$ cells per well and cultivated overnight. Following centrifugation at $1000 \mathrm{rpm}$ for $5 \mathrm{~min}$ at room temperature, culture medium was removed and cells were loaded with JC-1 dye for half an hour. After washing, the change in mitochondrial transmembrane potential $\left(\Delta \Psi_{\mathrm{m}}\right)$ was detected with a fluorescent plate reader. Images were captured by a fluorescence microscope.

For TUNEL staining assay, cells were transfected and cultivated all night. After rinsing twice in phosphatebuffered saline (PBS), cells were fixed in $4 \%$ paraformaldehyde for $15 \mathrm{~min}$ and permeabilized in $0.25 \%$ Triton-X 100 for $20 \mathrm{~min}$. TUNEL staining method was used following the guidelines of In Situ Cell Death Detection Kit (Roche Molecular Diagnostics, Pleasanton, CA, USA). Cell nuclei were treated with DAPI. Positively stained cells were observed under an EVOS FL microscope (Thermo Fisher Scientific, Waltham, MA, USA).

For detection of caspase-3 activity, caspase-3 activity kit was bought from Solarbio (Beijing, China). Cell proteins were extracted and seeded into 96-well plates. Afterwards, proteins were incubated with caspase-3 substrate for $3 \mathrm{~h}$.
The absorbance was detected at the wavelength of $405 \mathrm{~nm}$. All results of apoptotic assay were obtained from three different replications.

\section{In vivo tumorigenesis assay}

Male nude mice, aged $\sim 6$ weeks, were kept in an SPFgrade pathogen-free animal laboratory. This animal study had obtained the approval of the Animal Research Ethics Committee of the Second Affiliated Hospital Zhejiang University School of Medicine. A total of $5 \times 10^{6} \mathrm{LNCaP}$ cells were transfected and subcutaneously injected around the left flank of the nude mice (five mice per group). Tumor volume was examined as $0.5 \times$ length $\times$ width $^{2}$ every 7 days. Four weeks later, mice were killed, tumors were excised and weighed for further study.

\section{Microarray analysis}

Cells were transfected with shRNAs against IDH1-AS1 or negative control. Total RNA was extracted after $48 \mathrm{~h}$ transfection. Gene expression microarray analysis (Agilent Technologies, Santa Clara, CA, USA) was carried out as per the user guide. One hundred nanograms of total RNA was amplified and labeled by the Low-input Quick Amp Labeling Kit One-color (Agilent Technologies). The synthesized cRNA was hybridized to the SurePrint G3 Human GE microarray v2 (G4851; Agilent Technologies). Finally, the differentially expressed mRNAs were identified with the cut-off criteria of $P<0.05$ and fold-change $>2$. In our present study, Gene ontology (GO) analysis was performed based on the GO database (http://www. geneontology.org/). $P<0.05$ was considered to indicate statistical significance.

\section{Western blot assay}

Cell protein samples were extracted using RIPA buffer reagent (Thermo Fisher Scientific) and separated using 12\% SDS PAGE gels. Following transferring onto polyvinylidene fluoride membranes, bovine serum albumin (5\%) solution (Beyotime, Guangzhou, China) was used to block membranes for $2 \mathrm{~h}$ at room temperature. Primary antibodies, including anti-LC3 I/II (ab128025, Abcam, Cambridge, USA), anti-p62 (ab56416, Abcam), antiBeclin1 (ab210498, Abcam), anti-ATG5 (ab228668, Abcam), and anti-GAPDH (ab181602, Abcam), were incubated with membranes at $4{ }^{\circ} \mathrm{C}$ all night. Secondary antibodies were cultured with membranes at room temperature for $2 \mathrm{~h}$. At length, bound proteins were quantified using ECL Prime Western Blotting Detection reagent (GE Healthcare, Chicago, IL, USA). All experimental procedures were repeated for more than two times.

\section{Immunofluorescence assay}

$\mathrm{VCaP}$ and $\mathrm{LNCaP}$ cell lines were plated on culture slides until adherent to the slides. After rising in PBS twice, cells 
were fixed in ice-cold methanol-acetone for $10 \mathrm{~min}$ and sealed in 5\% BSA for $10 \mathrm{~min}$. Subsequently, cells were cultured with the primary antibodies against LC3 for $2 \mathrm{~h}$ at room temperature and rinsed in PBS for three times. Then, cells were incubated with secondary antibodies for $1 \mathrm{~h}$. After staining with DAPI for $10 \mathrm{~min}$, the slides from three replications were observed using a fluorescence microscope (Olympus, Tokyo, Japan).

\section{MDC staining assay}

$\mathrm{VCaP}$ and LNCaP cell lines were transfected and seeded in six-well plates for 3 days and rinsed for three times in PBS. Monodansylcadaverine solution (MDC; SigmaAldrich) was diluted to $1: 10$ and added staining solution to cover cells. Three days later, culture medium was discarded, the plates were washed twice with $300 \mu$ wash buffer. Then, cells were mixed with $50 \mu \mathrm{mol} / \mathrm{l}$ at $37^{\circ} \mathrm{C}$ for $1 \mathrm{~h}$ in the dark. Following fixation in $4 \%$ paraformaldehyde for $15 \mathrm{~min}$, cells were washed twice in cold PBS and photographed by fluorescence photometry. Three different experimental results were determined at $512 \mathrm{~nm}$.

\section{RNA immunoprecipitation (RIP) assay}

For RNA-binding protein immunoprecipitation assay, EZ-Magna RIP kit (Millipore) was utilized in line with the guidelines supplied by the manufacturer. Cell lysates were cultured with RIP buffer containing magnetic beads conjugated to human anti-PTBP3 antibody (Cell Signaling Technology, Danvers, MA) or negative control normal mouse IgG antibody (Millipore, CA, USA). AntiSNRNP70 antibody was seen as the positive control. Lysates were treated with Proteinase $\mathrm{K}$ and finally subjected to silver staining and qRT-PCR analysis. For Ago2RIP assay, cells were incubated with protein A/G sepharose beads conjugated to antibodies against Ago2 (Millipore, Massachusetts, USA) or IgG. Immunoprecipitated RNAs were extracted for qRT-PCR. Experiments were performed in triplicate.

\section{Subcellular fractionation assay}

Cytoplasmic and nuclear RNA were isolated based on the user guide of PARIS ${ }^{\text {mi }}$ Kit (Invitrogen, USA). A total of $1.0 \times 10^{7} \mathrm{VCaP}$ and LNCaP cell lines were lysed in cell fractionation buffer and incubated on ice for $10 \mathrm{~min}$. After centrifugation, the upper solution was discarded, the nuclear pellet was collected to extract RNA in cell disruption buffer. Biological triplicates were carried out and followed by qRT-PCR to detect RNA abundance.

\section{Statistical analysis}

All statistical analysis was conducted by SPSS version 20.0 software (IBM Corp., Armonk, NY, USA). Values were presented as the mean \pm standard deviation. $P$-value threshold was set as 0.05 to indicate a statistically significant difference. Statistical comparisons were carried out using the Student's $t$ test or one-way analysis of variance followed by the Dunnett's test. Gene correlations were analyzed by the Pearson's correlation method.

\section{Results}

IDH1-AS1 is upregulated in PCa tissues and cell lines

According to the gene expression profile in GEPIA database, IDH1-AS1 is a highly expressed lncRNA in PCa samples (Fig. 1a). Sixty-two PCa patients were recruited in this study. And these patients were divided into two groups in accordance with tumor stage (I/II and III/IV). IDH1-AS1 expression was detected in different tissues collected from these patients. After qRT-PCR exanimation, we determined that IDH1-AS1 was expressed higher in tumor tissues and advanced stage patient samples compared with normal tissues and early-stage patient samples (Fig. 1b, c). Consistently, IDH1-AS1 expressed at a higher level in PCa cell lines compared with normal epithelial cell line (Fig. 1d). These data suggested that IDH1-AS1 potentially participated in tumorigenesis of PCa.

\section{IDH1-AS1 is transcriptionally activated by PAX5 in PCa}

Transcription activation is one of the main molecular mechanisms which led to the upregulation of genes. Screening from UCSC online database, PAX5 is a potential upstream transcription regulator for IDH1-AS1. According to qRT-PCR analysis, PAX5 was identified to upregulated in $62 \mathrm{PCa}$ samples (Fig. 2a), which had a positive expression correlation with IDH1-AS1 (Fig. 2b). Similarly, PAX5 was also expressed at a relative high level in PCa cell lines (Fig. 2c). To assess whether PAX5 had regulatory effect on IDH1-AS1 expression, we silenced or overexpressed it in LNCaP and VCaP cells (Fig. 2d). Then, we examined the expression change of IDH1-AS1 in above cell lines and uncovered the positive regulatory effect of PAX5 on IDH1-AS1 expression in PCa cells (Fig. 2e). DNA binding motif of PAX5 was obtained from JASPAR (Fig. 2f) and was subjected to the prediction of binding sites with IDH1-AS1 promoter. There existed a binding sequence between PAX5 and IDH1-AS1 promoter (Fig. 2g). Next, pGL3 luciferase reporter assay was conducted to demonstrate whether the binding sequence was responsible for the interaction between PAX5 and IDH1-AS1 promoter. Data presented in Fig. 2h, the luciferase activity of IDH1-AS1 promoter was increased after overexpression of PAX5 but was reduced in cells transfected with shPAX5. Furthermore, we found that PAX5 had a strong affinity in the promoter region of IDH1-AS1 through ChIP assay (Fig. 2i). Collectively, upregulation of IDH1-AS1 in LUAD cell lines might be caused by PAX5-induced transcription activation. 

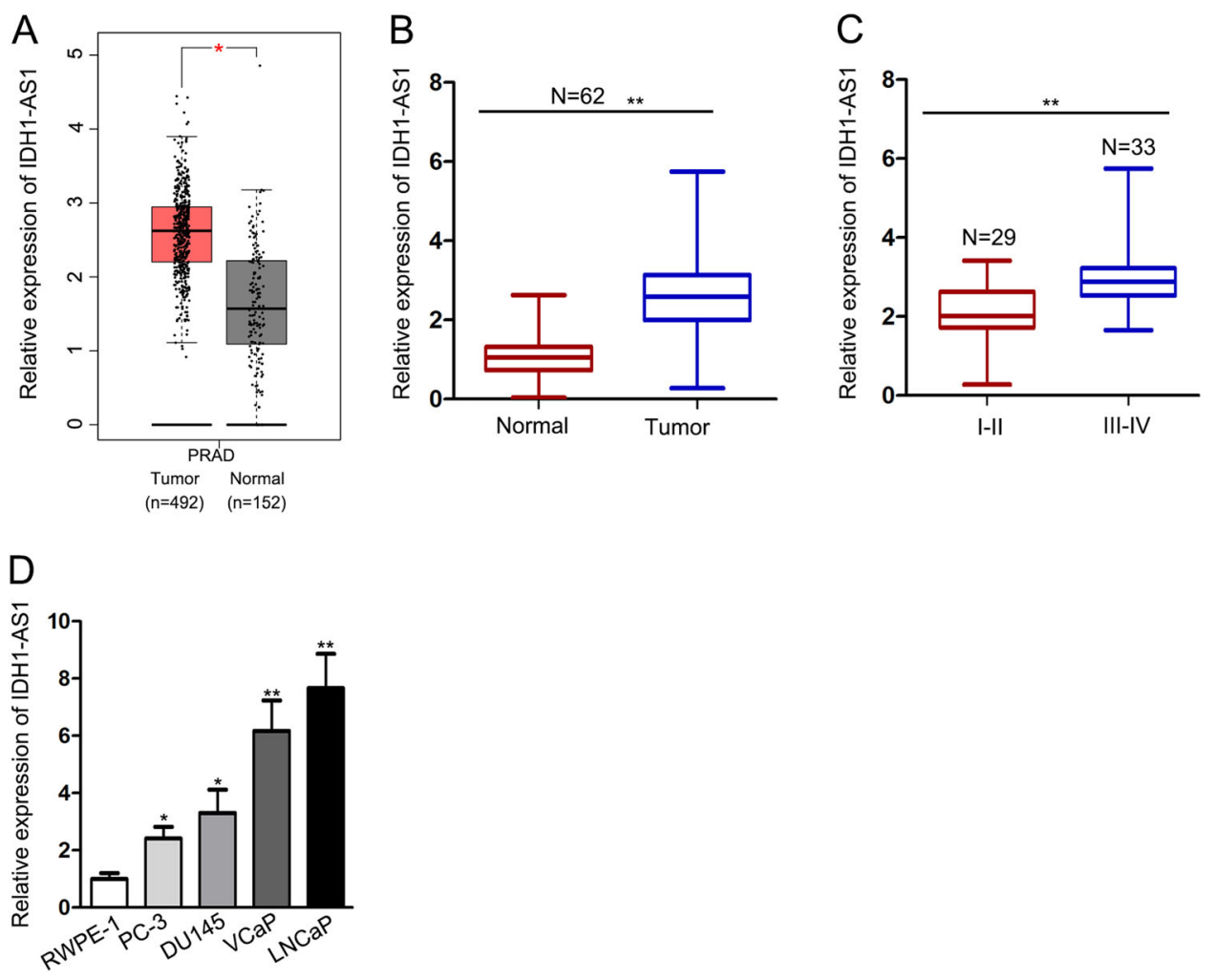

Fig. 1 IDH1-AS1 is upregulated in PCa tissues and cell lines. a IDH1-AS1 expression level in PCa samples obtained from GEPIA database. b qRTPCR showing IDH1-AS1 expression in paired PCa and normal samples collected from 62 PCa patients. c Expression level of IDH1-AS1 in PCa tissues in early stage of advanced stage was measured using qRT-PCR analysis. $\mathbf{d}$ IDH1-AS1 expression level was examined in PCa cell lines and normal epithelial cell line. ${ }^{*} P<0.05,{ }^{* *} P<0.01$

\section{IDH1-AS1 acts as a facilitator for PCa cell growth in vitro and in vivo}

Based on findings above, we determined the upregulation of IDH1-AS1 in PCa and its upregulatory mechanism. Considering the highest expression level of IDH1AS1 in VCaP and LNCaP cells, we conducted loss-offunction studies in these two cells. Silencing of IDH1-AS1 by shRNAs was determined by qRT-PCR analysis (Supplementary Fig. 1a). At first, we evaluated the effect of silenced IDH1-AS1 on cell proliferation. Through cell proliferation assays, including CCK-8, colony formation and EdU assays, we uncovered that cell proliferation was attenuated in response to the downregulation of IDH1AS1 (Fig. 3a-c). In addition, apoptosis was measured in transfected $\mathrm{PCa}$ cells via JC-1 and TUNEL assays. As expected, suppression of IDH1-AS1 expression accelerated apoptosis (Fig. 3d, e). For further evidence, animal study was carried out. After 28 days, tumors were removed from the body of nude mice. After observation and calculation, we identified that tumors in the shIDH1AS1 group grew smaller than those in the shCtrl group (Supplementary Fig. 1b). This tendency was further validated in tumor volume and weight (Supplementary
Fig. 1c, d). Based on all these data, IDH1-AS1 was identified as a tumor facilitator in PCa.

\section{IDH1-AS1 regulated ATG5-mediated autophagy in PCa}

Subsequently, we analyzed IDH1-AS1-dominated molecular mechanism in PCa. Through microarray analysis, we screened out top 200 mRNAs that were significantly downregulated in IDH1-AS1-downregulated cells (Fig. 4a). Then, these 200 mRNAs were subjected to GO analysis. As presented in Fig. 4b, IDH1-AS1dominated molecular mechanism potentially mediated cell apoptosis and autophagy. Importantly, ATG5, a biomarker for autophagy, belongs to top 200 downregulated mRNAs. Using qRT-PCR examination, we further demonstrated the positive regulation of IDH1-AS1 on ATG5 in two PCa cells (Fig. 4c). Thus, we further detected whether IDH1-AS1 regulated ATG5-mediated autophagy in PCa cells. The level of autophagy-related proteins, LC3 fluorescence intensity, and the ratio of autophagosome were detected successively. All experimental results indicated that IDH1-AS1 acted as an inducer in autophagy of PCa cells (Fig. 4d, f). Finally, ATG5 expression was uncovered to be higher in $\mathrm{PCa}$ 


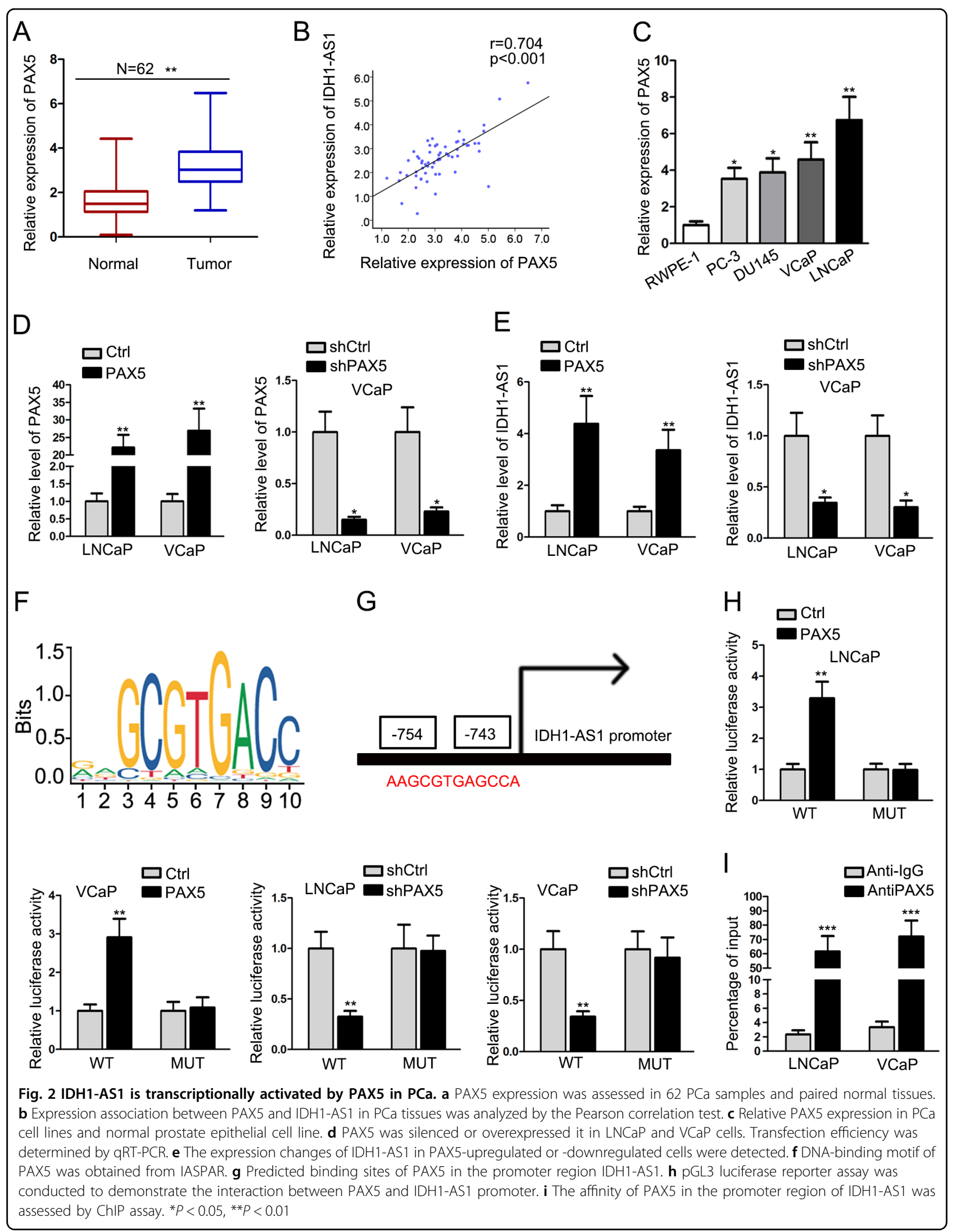




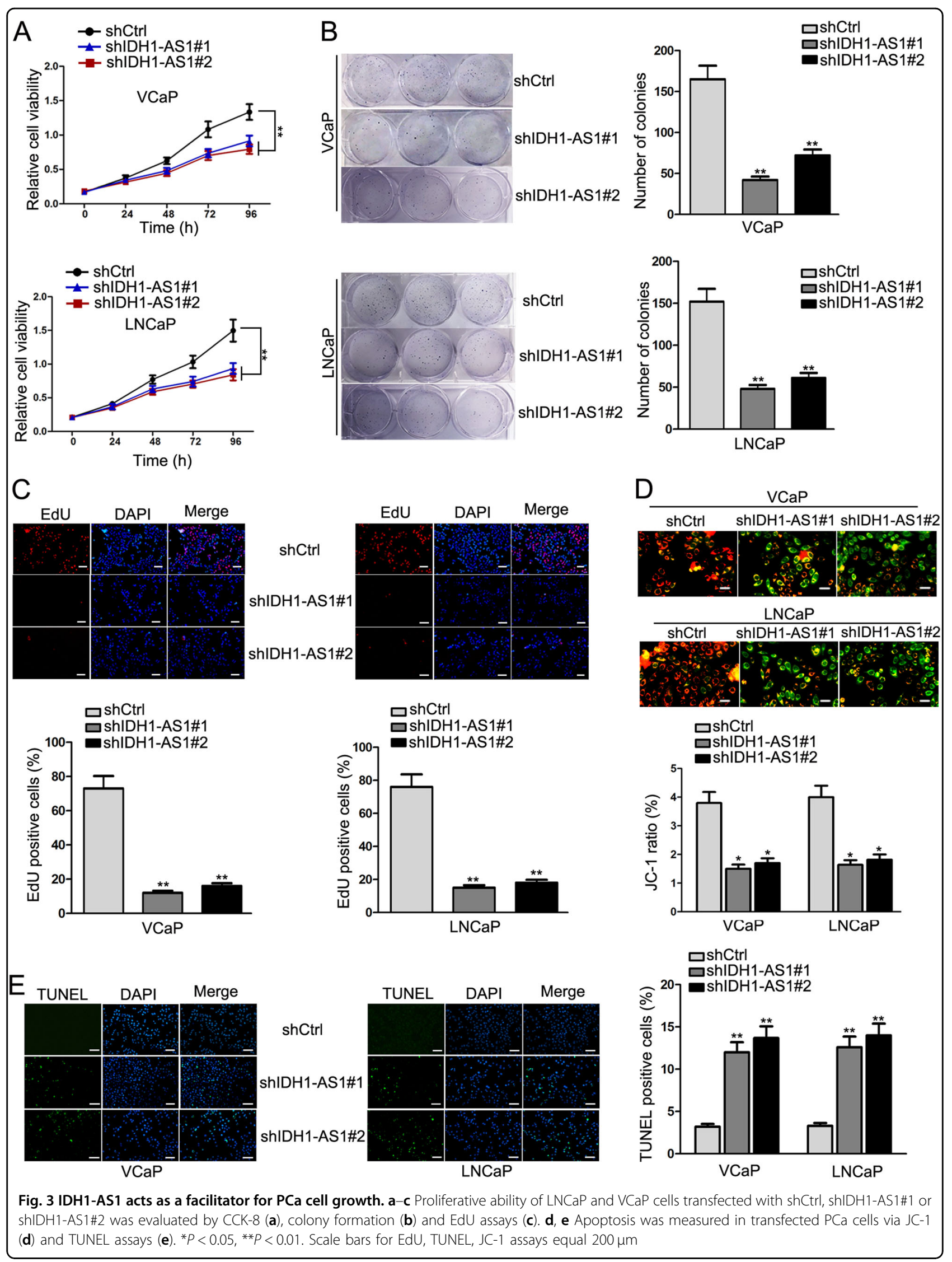




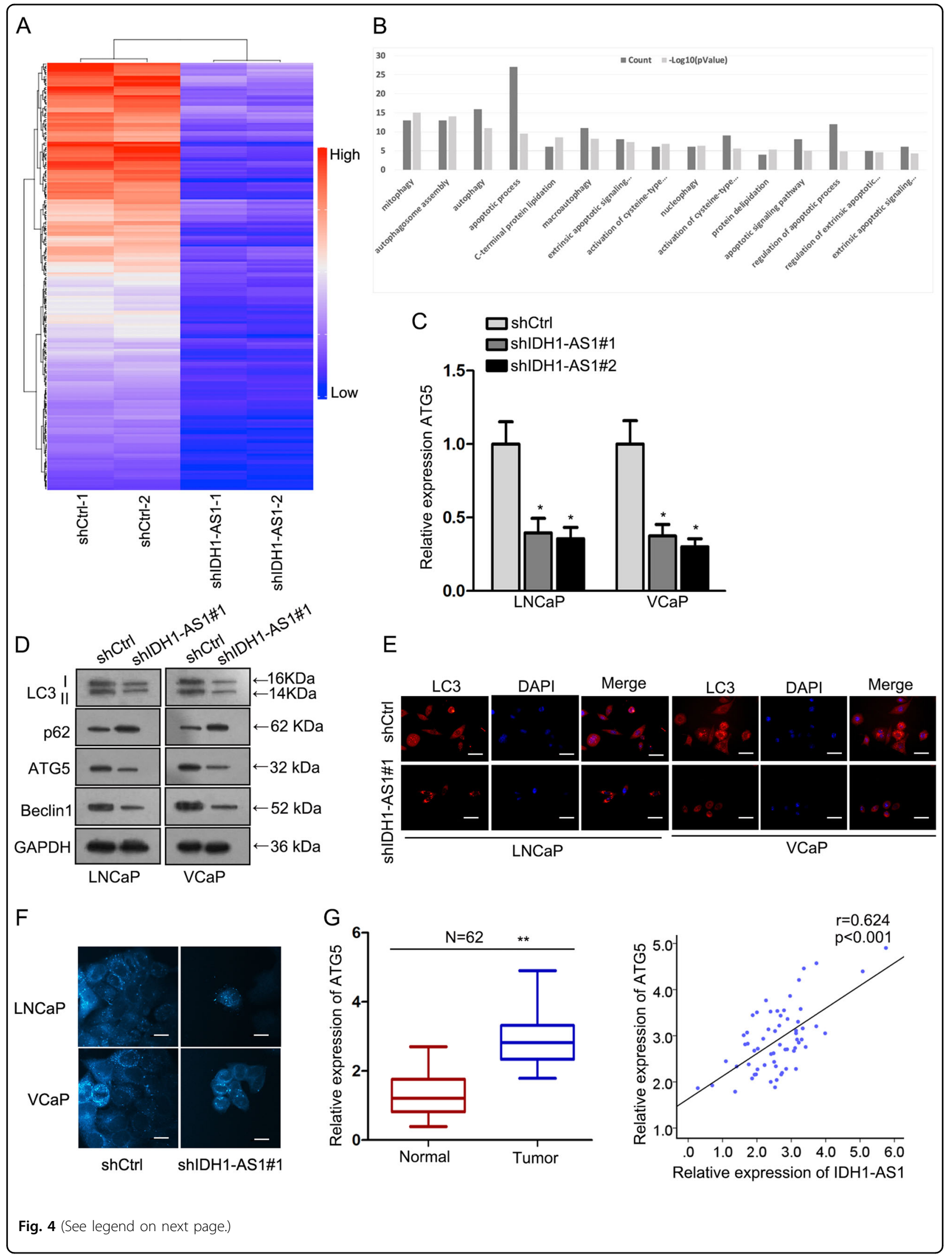


(see figure on previous page)

Fig. 4 IDH1-AS1 regulated ATG5-mediated autophagy in PCa. a Top 200 mRNAs that were significantly downregulated in IDH1-AS1-silenced cells were screened out by microarray analysis. b GO analysis of IDH1-AS1-reulated 200 mRNAs. c ATG5 expression level in cells transfected with IDH1-AS1-specific shRNAs and control shRNA was measured using qRT-PCR examination. The level of autophagy-related proteins, LC3 fluorescence intensity and the ratio of autophagosome were detected successively by western blotting (d), LC3 immunofluorescence (e), and MDC staining (f). g ATG5 expression in PCa tissues and its expression association with IDH1-AS1 expression. ${ }^{*} P<0.05$, ${ }^{*} P<0.01$. Scale bars for LC3 immunofluorescence and MDC staining equal $200 \mu \mathrm{m}$

tissues than in normal tissues, which had a consistent tendency with IDH1-AS1 expression (Fig. 4g). Collectively, IDH1-AS1 might regulate autophagy through ATG5.

\section{IDH1-AS1 interacted with PTBP3 to stabilize ATG5 mRNA}

To determine the regulatory pattern of IDH1-AS1 in PCa cells, we analyzed its cellular localization. The results shows in Supplementary Fig. 1e indicated that IDH1-AS1 was predominantly located in cytoplasm of PCa cells. Results of pGL3 luciferase reporter assay revealed that there was no significant effect of IDH1-AS1 on the luciferase activity of ATG5 promoter (Supplementary Fig. 1f), which eliminated the transcriptional regulation of IDH1AS1 on ATG5. Through RNA pull-down and massspectrometry analysis, we found that PTBP3 is a RBP which could interact with IDH1-AS1 (Fig. 5a). The interaction between PTBP3 and IDH1-AS1 or ATG5 was further proved by RIP assay (Fig. 5b). Then, we overexpressed PTBP3 in LNCaP and VCaP cell and found that ATG5 expression was observably increased (Fig. 5c, d). Subsequently, ATG5 mRNA stability was assessed in PCa cells treated with Actinomycin D after overexpression of PTBP3. It was found that PTBP3 overexpression enhanced the stability of ATG5 (Fig. 5e). Furthermore, we analyzed whether IDH1-AS1 affected the interaction between PTBP3 and ATG5. As a result, silencing of IDH1-AS1 attenuated the interaction between PTBP3 and ATG5 (Fig. 5f). Accordingly, knockdown of IDH1AS1 impeded the mRNA stability of ATG5, which tendency was attenuated after co-transfection with PTBP3 expression vector (Fig. 5g). Taken together, IDH1-AS1 regulated ATG5 mRNA stability via interacting with PTBP3.

\section{IDH1-AS1 upregulated ATG5 expression by sponging miR- 216b-5p}

CeRNA regulatory network is an important posttranscriptional regulatory mechanism of lncRNAs. Here, we also explored whether IDH1-AS1 and ATG5 could form a ceRNA pathway. Bioinformatics analysis suggested that miR-216b-5p had complementary base pairing with both IDH1-AS1 and ATG5. To validate their interaction, IDH1-AS1 or ATG5 3'UTR containing the binding sequences with miR-216b-5p was cloned into luciferase reporter vectors. It was uncovered that the luciferase activity of wild type reporters was decreased by miR-216b$5 \mathrm{p}$ mimics, whereas no obvious changes in mutant reporters (Fig. 6a, b). Then, we examined the mRNA and protein level of ATG5 in cells transfected with miR-NC or miR-216b-5p mimics. The levels of ATG5 were significantly impaired by the upregulation of miR-216b-5p (Fig. 6c). Ago2-RIP assay was used to prove the enrichment of IDH1-AS1, miR-216b-5p, and ATG5 in RISC complex (Fig. 6d). Afterwards, miR-216b-5p expression was found to be lower in PCa tissues than that in normal controls (Fig. 6e). Pearson correlation analysis revealed that miR-216b-5p had negative expression correlation with IDH1-AS1 and ATG5 (Fig. 6f). All these experimental results indicated that IDH1-AS1 functioned as a ceRNA to regulate ATG5 expression via sponging miR216b-5p.

\section{IDH1-AS1 promoted PCa cell growth by regulating ATG5- mediated autophagy}

To demonstrate IDH1-AS1 promoted PCa cell growth via ATG5, rescue assays were carried out in LNCaP cells. Through cell proliferation assay, we determined that co-transfection with ATG5 expression vector rescued shIDH1-AS1-induced proliferation-inhibition (Fig. 7a-c). Moreover, shIDH1-AS1-induced apoptosis was reduced after overexpression of ATG5 (Fig. 7d, e). Simultaneously, changes in autophagy were observed. It was showed that ATG5 overexpression recovered the autophagy suppressed by IDH1-AS1 knockdown (Fig. 7f, g). Combining with all experimental data above, PAX5-activated IDH1-AS1 acted as an oncogene in PCa tumor growth by promoting ATG5-induced autophagy (Fig. 8).

\section{Discussion}

Recent years, lncRNAs have been elucidated in various reports due to their biological functions ${ }^{21-23}$. In addition, dysregulation of lncRNAs is acknowledged as an important factor for tumorigenesis ${ }^{24-26}$. The key finding of current study is that IDH1-AS1 was upregulated in PCa tissues and cells. In our current study, we investigated whether IDH1-AS1 is a regulator in PCa tumorigenesis. 


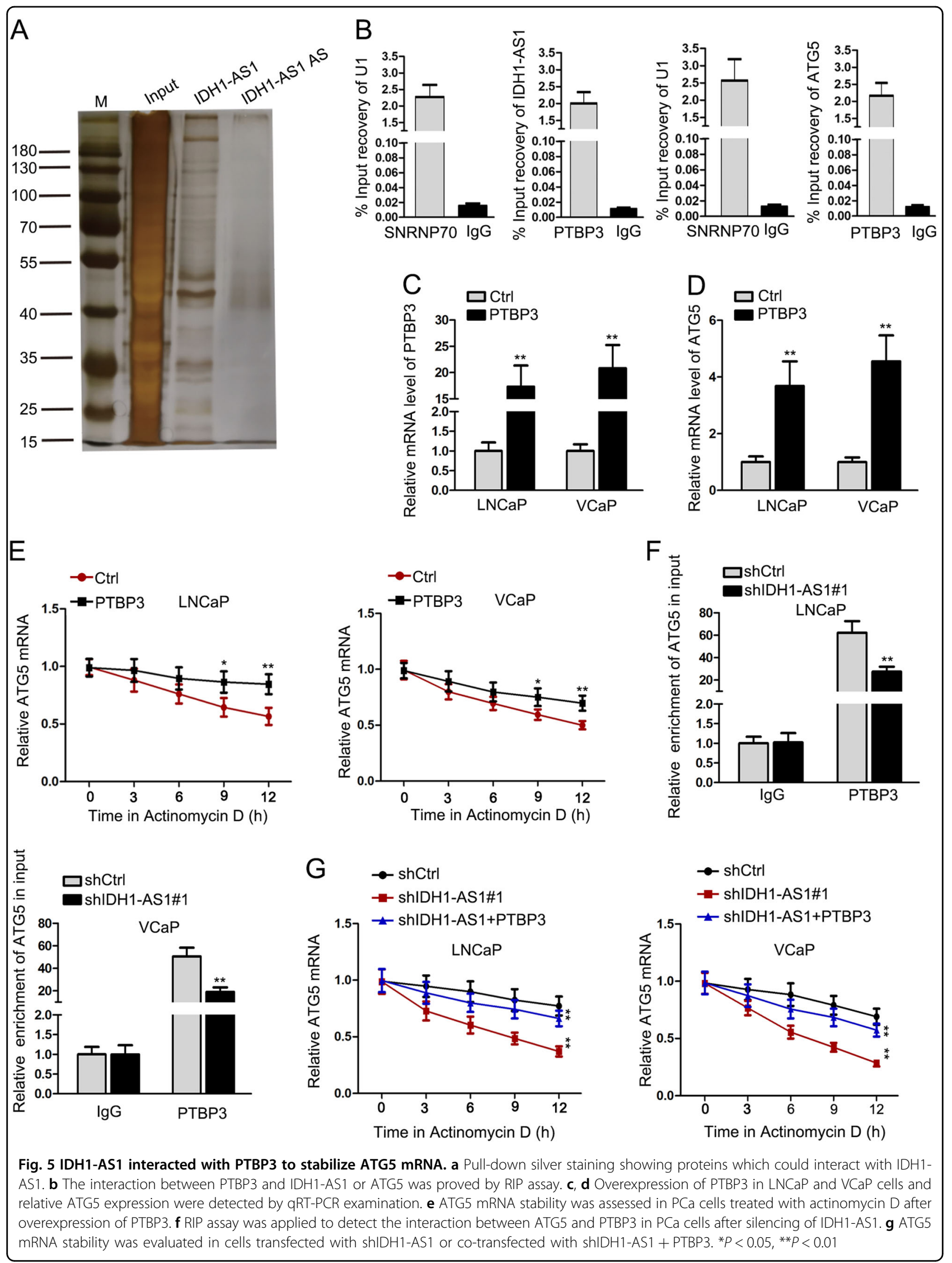




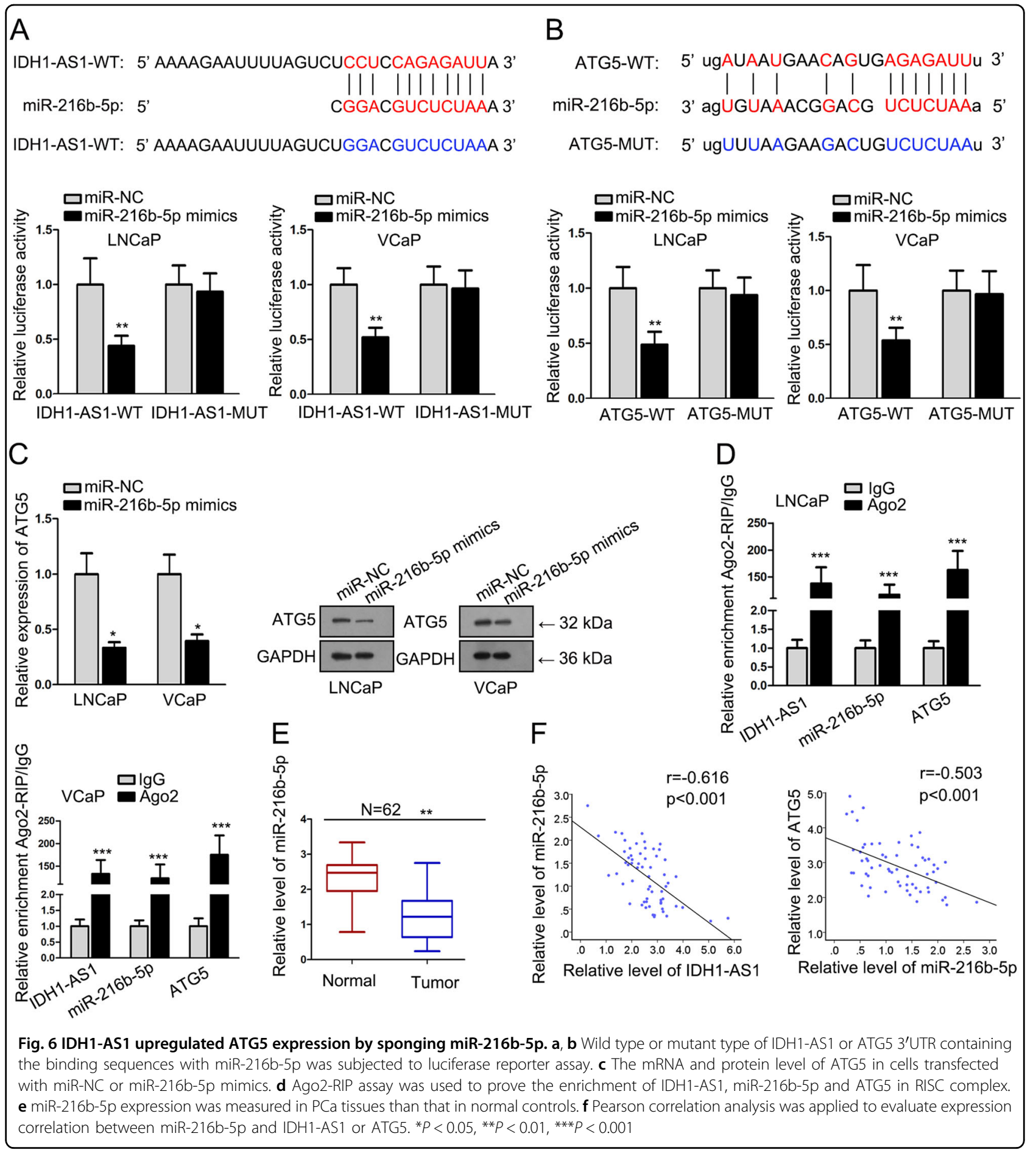

Through loss-of function assays, we determined that IDH1-AS1 had a positive effect on PCa cell proliferation but a negative effect on PCa cell apoptosis. Moreover, animal study was carried out and proved that IDH1-AS1 was a facilitator for $\mathrm{PCa}$ tumor growth. Therefore, oncogenic property of IDH1-AS1 in PCa tumorigenesis was identified in this study.
Transcription activation is a reason for the upregulation of lncRNAs in tumorigenesis ${ }^{11-13}$. In this study, we analyzed the upstream molecular mechanism of IDH1-AS1. Through mechanism investigation, PAX 5 was verified to be a transcription activator for IDH1-AS1 and positively regulated IDH1-AS1 expression in PCa. Downstream molecular mechanism of IDH1-AS1 in PCa was further 

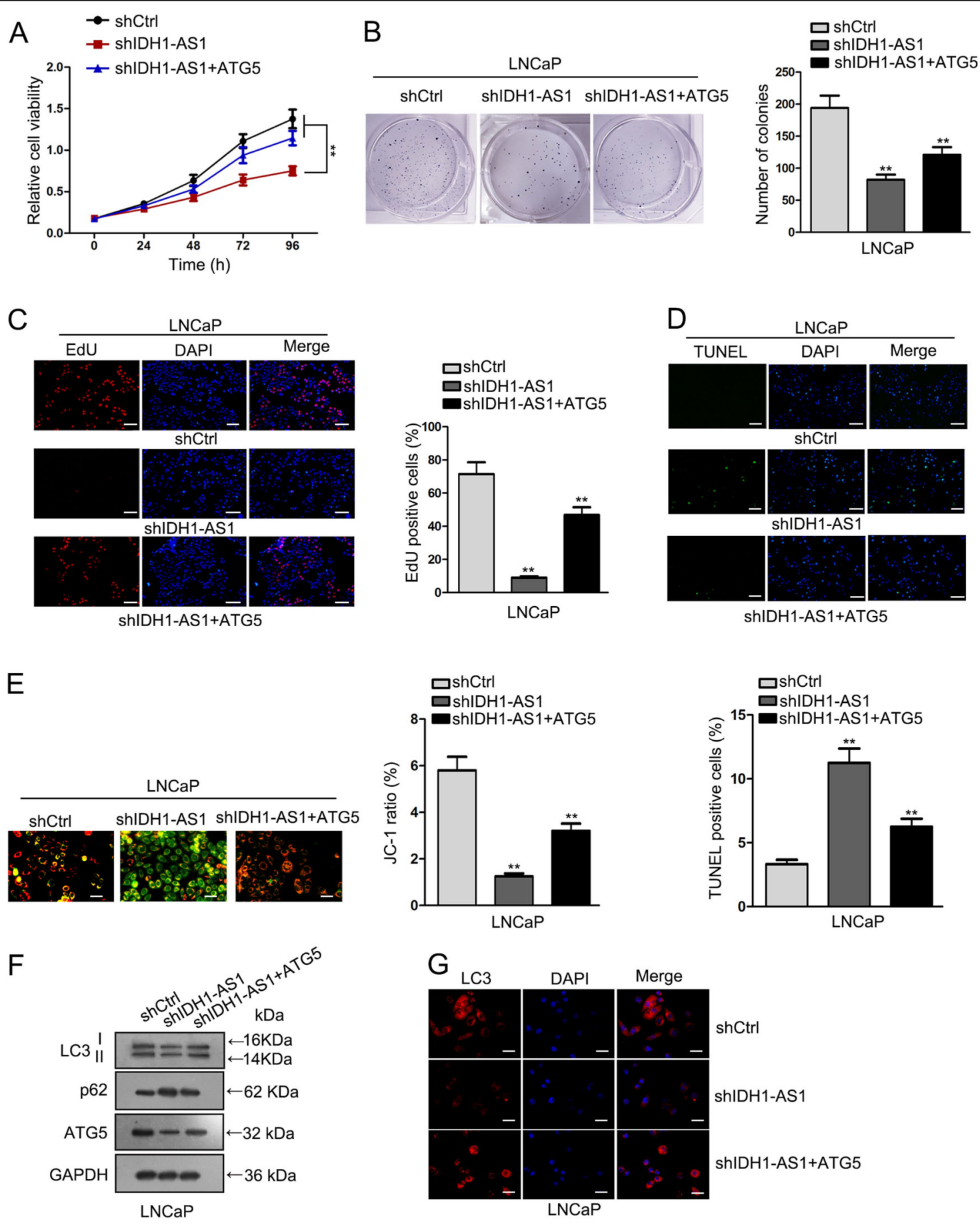

Fig. 7 IDH1-AS1 promoted PCa cell growth by regulating ATG5-mediated autophagy. The involvement of ATG5 in IDH1-AS1-mediated cell proliferation was determined by CCK-8 (a), colony formation (b) and EdU assays (c). Apoptosis was measured in indicated PCa cells through JC-1 (d) and TUNEL assays (e). f, $\mathbf{g}$ Changes in autophagy were observed in indicated PCa cells by western blotting and LC3 immunofluorescence test. ${ }^{*} P<0.01$. Scale bars for EdU, TUNEL, JC-1 assays and LC3 immunofluorescence equal $200 \mu \mathrm{m}$

investigated. Through microarray analysis and GO analysis, we uncovered that IDH1-AS1 potentially regulated autophagy in PCa cells via modulating ATG5 expression. According to the experimental results, we determined that IDH1-AS1 promoted autophagy in PCa cells by upregulating ATG5.

Based on all findings above, we continued to explore whether IDH1-AS1 exerts functions by regulating ATG5 


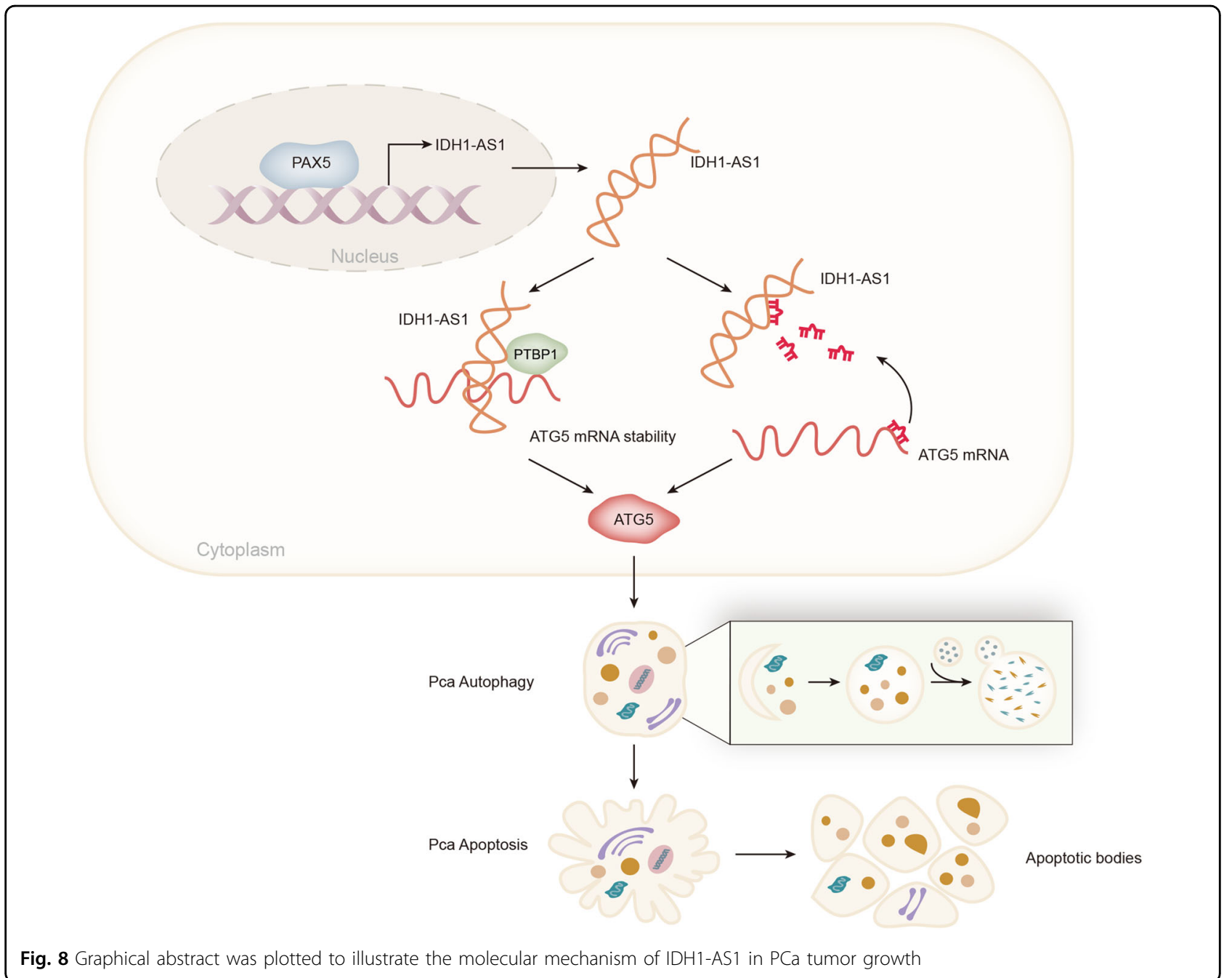

expression. Luciferase activity of ATG5 promoter was not affected by IDH1-AS1 and the cytoplasmic localization of IDH1-AS1 prompted us to investigate posttranscriptional regulation of IDH1-AS1 on ATG5. There are two regulatory patterns of lncRNAs at post-transcriptional level. For example, IncRNAs can regulate mRNA stability by interacting with $\mathrm{RBPs}^{17-20}$; lncRNAs positively regulate mRNAs by sequestering miRNAs ${ }^{14-16}$. At first, pull-down silver staining followed by mass-spectrometry analysis revealed that there were some RBPs that could interact with IDH1-AS1. Mechanism investigation showing the interactions between IDH1-AS1 and PTBP3 as well as between PTBP3 and ATG5. Furthermore, we found that IDH1-AS1 enhanced the stability of ATG5 mRNA by cooperating with PTBP3. Subsequently, we investigated whether IDH1-AS1 acts as a ceRNA to regulate ATG5 in PCa cells. Similarly, mechanism study demonstrated that IDH1-AS1 acted as a ceRNA to upregulate ATG5 expression by sequestering miR-216b-5p. At length, we analyzed the involvement of ATG5 in IDH1-AS1mediated cell growth and autophagy by rescue assays. It was uncovered that cell growth and autophagy that were suppressed by IDH1-AS1 knockdown were recovered by the overexpression of ATG5. Taken together, we confirmed that IDH1-AS1 acted as a growth facilitator in PCa cells via regulating ATG5-mediated autophagy.

In conclusion, this study reported a novel molecular pathway in PCa tumorigenesis. Upregulation of IDH1AS1 was induced by PAX5 transcription activator. Moreover, upregulation of IDH1-AS1 facilitated PCa tumor growth by posttranscriptionally regulating ATG5 expression. All findings in this study may contribute to unveil molecular mechanism associated with tumorigenesis of $\mathrm{PCa}$, thus providing new potential diagnostic or therapeutic biomarkers.

\section{Acknowledgements}

The authors sincerely appreciate members contributed to this study. 


\section{Funding}

This study was supported by Zhejiang Natural Science Foundation (LY19H050006 and LY18H050002); Zhejiang Science and Technology Department Public Welfare Project (2017C33063).

\section{Conflict of interest}

The authors declare that they have no conflict of interest.

\section{Publisher's note}

Springer Nature remains neutral with regard to jurisdictional claims in published maps and institutional affiliations.

Supplementary Information accompanies this paper at (https://doi.org/ 10.1038/s41419-019-1932-3).

Received: 24 June 2019 Revised: 6 August 2019 Accepted: 20 August 2019 Published online: 30 September 2019

\section{References}

1. Jemal, A. et al. Global cancer statistics. CA: Cancer J. Clinicians 61, 69-90 (2011).

2. Prensner, J. R. \& Chinnaiyan, A. M. The emergence of IncRNAs in cancer biology. Cancer Discov. 1, 391-407 (2011).

3. Kapranov, P. et al. RNA maps reveal new RNA classes and a possible function for pervasive transcription. Science 316, 1484-1488 (2007).

4. Huarte, M. \& Rinn, J. L. Large non-coding RNAs: missing links in cancer? Hum. Mol. Genet. 19, R152-161 (2010).

5. Liu, Q. et al. LncRNA loc285194 is a p53-regulated tumor suppressor. Nucleic Acids Res. 41, 4976-4987 (2013).

6. Peng, L. et al. Super-enhancer-associated long non-coding RNA HCCL5 is activated by ZEB1 and promotes the malignancy of hepatocellular carcinoma. Cancer Res. https://doi.org/10.1158/0008-5472.can-18-0367 (2018).

7. Fang, Z. et al. LncRNA UCA1 promotes proliferation and cisplatin resistance of oral squamous cell carcinoma by sunppressing miR-184 expression. $\mathbf{6}$, 2897-2908, https:/doi.org/10.1002/cam4.1253 (2017).

8. Gibb, E. A., Brown, C. J. \& Lam, W. L. The functional role of long non-coding RNA in human carcinomas. Mol. Cncer 10, 38 (2011).

9. Shang, Z. et al. LncRNA PCAT1 activates AKT and NF-kappaB signaling in castration-resistant prostate cancer by regulating the PHLPP/FKBP51/ IKKalpha complex. Nucleic Acids Res. https://doi.org/10.1093/nar/gkz108 (2019).

10. Xiang, S., Gu, H. \& Jin, L. LncRNA IDH1-AS1 links the functions of c-Myc and HIF1alpha via IDH1 to regulate the Warburg effect. 115, E1465-E1474, https:// doi.org/10.1073/pnas.1711257115 (2018).
11. Liu, H. T., Liu, S., Liu, L., Ma, R. R. \& Gao, P. EGR1-mediated transcription of IncRNA-HNF1A-AS1 promotes cell-cycle progression in gastric cancer. Cancer Res. 78, 5877-5890 (2018).

12. Xu, M. D. et al. A positive feedback loop of IncRNA-PVT1 and FOXM1 facilitates gastric cancer growth and invasion. Clin. Cancer Res. 23, 2071-2080 (2017).

13. Chen, X. et al. SP1-induced IncRNA-ZFAS1 contributes to colorectal cancer progression via the miR-150-5p/NEGFA axis. 9, 982, https://doi.org/10.1038/ s41419-018-0962-6 (2018).

14. Yan, X. et al. Mesenchymal stem cells promote hepatocarcinogenesis via IncRNA-MUF interaction with ANXA2 and miR-34a. Cancer Res. https://doi.org/ 10.1158/0008-5472.can-17-1915 (2017).

15. Chen, X. et al. Long noncoding RNA LINC01234 functions as a competing endogenous RNA to regulate CBFB expression by sponging miR-204-5p in gastric cancer. Clin. Cancer Res. https://doi.org/10.1158/1078-0432.ccr-17-2376 (2018).

16. Chen, D. L. et al. Long non-coding RNA UICLM promotes colorectal cancer liver metastasis by acting as a ceRNA for microRNA-215 to regulate ZEB2 expression. Theranostics 7, 4836-4849 (2017).

17. Wang, A. et al. Long noncoding RNA EGFR-AS1 promotes cell growth and metastasis via affecting HuR mediated mRNA stability of EGFR in renal cancer. Cell Death Dis. 10, 154 (2019).

18. Xiao, Y., Pan, J., Geng, Q. \& Wang, G. LncRNA MALAT1 increases the stemness of gastric cancer cells via enhancing SOX2 mRNA stability. FEBS Open Bio. https://doi.org/10.1002/2211-5463.12649 (2019).

19. Wu, H. et al. LncRNA THOR increases osteosarcoma cell stemness and migration by enhancing SOX9 mRNA stability. FEBS Open Bio 9, 781-790 (2019).

20. Zhang, Y. et al. LBX2-AS1 is activated by ZEB1 and promotes the development of esophageal squamous cell carcinoma by interacting with HNRNPC to enhance the stability of ZEB1 and ZEB2 mRNAs. Biochem Biophys Res. Commun. 511, 566-572 (2019).

21. Jiang, L. et al. HCP5 is a SMAD3-responsive long non-coding RNA that promotes lung adenocarcinoma metastasis via miR-203/SNAl axis. Theranostics $\mathbf{9}$ 2460-2474 (2019)

22. Qin, G. et al. IncRNA PSTAR Promotes $p 53$ signaling by inhibiting hnRNP $K$ deSUMOylation and suppresses hepatocellular carcinoma. Hepatology. https:/ doi.org/10.1002/hep.30793 (2019).

23. Schmidt, K. et al. The IncRNA SLNCR recruits the androgen receptor to EGR1bound genes in melanoma and inhibits expression of tumor suppressor p21. Cell Rep. 27, 2493-2507.e2494 (2019).

24. Huang, J. F. et al. Genome-wide screening identifies oncofetal IncRNA Ptn-dt promoting the proliferation of hepatocellular carcinoma cells by regulating the Ptn receptor. Oncogene 38, 3428-3445 (2019).

25. He, F. et al. Long noncoding RNA PVT1-214 promotes proliferation and invasion of colorectal cancer by stabilizing Lin28 and interacting with miR-128. Oncogene 38, 164-179 (2019).

26. Kong, F. et al. ZFPM2-AS1, a novel IncRNA, attenuates the p53 pathway and promotes gastric carcinogenesis by stabilizing MIF. Oncogene 37, 5982-5996 (2018). 